\title{
Computational Experiments with the Fuzzy Love and Romance
}

\author{
Hokky Situngkir \\ hs@compsoc.bandungfe.net \\ Dept. Computational Sociology \\ Bandung Fe Institute
}

April $15^{\text {th }} 2007$

\begin{abstract}
The paper report some experiments on the issue of human mating games and sexual preferences in the perspective of population and some macro-social realms. The notions of love, romance, fidelity, and sexual attractiveness are those comprising the theory to human intra-species evolution but yet still rare to be employed to understand human social, economic, and cultural studies in terms of sociology or economics. The paper did experiments on those issues, on the possible relation between population growth, powerlaw distribution of wealth, and some other relevant points to our realization of evolutionary theory of sexual selection. The paper expects to open an alternative bridge of our advancement in human evolution and the complexity of the social system.
\end{abstract}

Keywords: human mating games, sexual selection, love and romance, macro-micro linkage, social simulation, artificial society. 
tria sunt difficilia mihi et quartum penitus ignoro: viam aquilae in caelo, viam colubri super petram, viam navis in medio mari, et viam viri in adulescentula

- Wisdom of King Solomon

\section{Intro}

At least our sights would have been divided into two scientific poles - both can be regarded in the domain of social science - as we talk about love and romance in human civilization. The first corner is laid upon the idea of sexual selection, latter called themselves as evolutionary psychologists. People in this pole are fascinated to reveal things behind behavioral aspects of the social life and pondering to the patterns discovered from surveys, behavioral analysis, and theoretical stuffs departed from sociobiology. The other pole is overwhelmed with people called themselves anthropologists, sociologists, and sometimes economists with very different views regarding to the relatedness of sex and the emotional feelings of love and romance. The latter mostly related the issue to the things that is constructed in social life through habits, norms, while the first mostly related it to the intraspecies evolution - thing that is so distinctive to its counterpart of inter-species evolution that apparently ruled by the law of struggle of the fittest by means of natural selection.

The history of the development of sociological theories has seen that there is not much endeavors to relate the findings in social and economic sciences with the one developed in natural science, especially biology. Despite the abundance of biologists' works with efforts to explaining some social processes - e.g.: evolutionary theories, memetics social scientists seem to neglect any theoretical achievements in other areas (cf. van den Berghe, 1990). As the advanced mathematical modeling of statistical mechanics has given a lot of contributions and stunning discoveries in economics (i.e.: econophysics, sociophysics), the advanced and powerful theoretical explorations in biology, especially in our understanding to the evolutionary concepts is felt highly important to be related to the discourse of the social dynamics (cf. Situngkir, 2003 and Situngkir, 2004).

The aim of the paper is to open the door to the new fashion of both theoretical and empirical approaches on bridging the most recent trend of sociological analyses incorporating computational models (i.e.: agent based modeling) to explore culture and social dynamics (even the embedded economic system) with some recent trends in evolutionary psychology with expectations of having novel discoveries in our scientific quests and inquiries to the general social life.

The structure of the paper is composed in regards to some experiments we do on the micro-states in the level of agency to see the emerging macro-properties. Our observations are made by using the artificial society the 'Ribbon of Love' as proposed in Situngkir (2007). We need to note here that the computational model we used here is different with some rigorous mathematical models constructed to see the interpersonal relations as those elaborated in Rinaldi (1998) or Liao \& Ran (2007) as introduced for educational purposes of differential equations by Strogatz (1988). Our model focuses not merely in interesting mathematical features of the loving couples but in deed focus on the non-linear relations of micro-social and its macro-view counterpart as a form of celebration in the new computational methods in the explorations of social theories. 


\section{Things can be observed in romantic population}

Interesting questions related to love and romance in the discussions of human interpersonality - whether it is evolutionary psychology or general social theories - are what construct our preferences over other persons and how they give impact to our social daily life. The relations of micro-preferences with things that we can see in macro-views are inherently non-linear and it is in some ways far from simplicity. The computational model introduced in previous work (Situngkir, 2007) somehow let us to do experiments and give tools to observe such non-linearity.
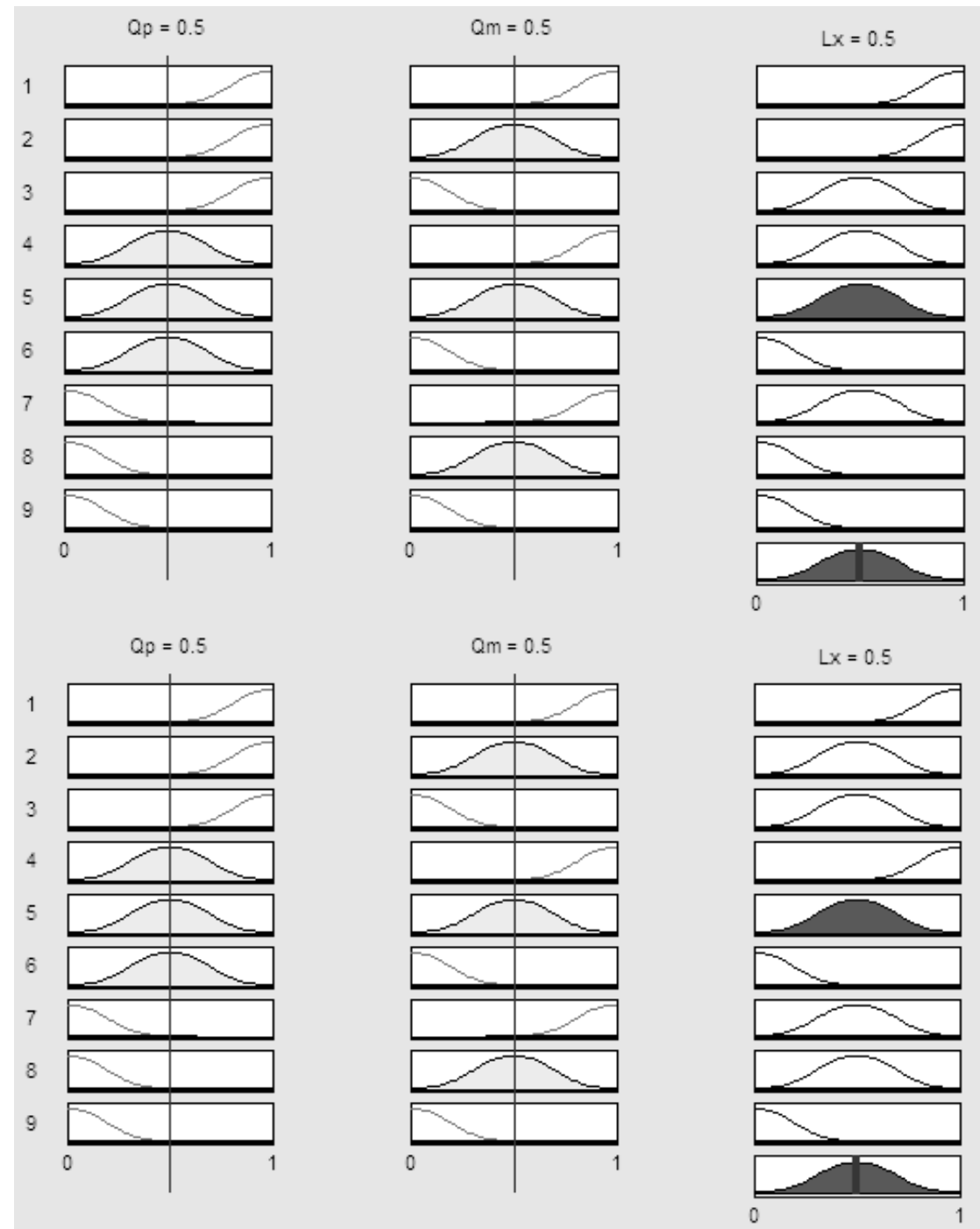

Figure 1

The rules depicting the loveable index of a female as seen by males in our artificial societies (above) and vice versa (below).

The fuzzy rules are as shown in figure 1 on male-to-female attraction and vice versa. The rules showed the micro-view of what females see in males and what attract males of females. However, agents are bounded in their neighborhood. Accessibility to preferred opposite sex is the most important constraint on how someone chooses the right mate. As 
accessibility is fulfilled, the next constraints are (in general) merely the sexual qualities emanate from the corresponding candidates. However, this kind of things has been recognized in social sciences as boundedness of a social actor with their neighbors. A social agent sociologically cannot be separated from the neighbors of which always to be referred when a decision is in making. In our model, such boundaries would be different when mating and how to choose the right one and the one when social choice is in the house. Furthermore, the size of neighbors for the reference of sexual relationship is made greater than the ones used in the way to higher the competitiveness of the same sex to attract any prospects of candidates.

Social sciences are interested most in the idea on how the heterogeneous microproperties of the interacting social actors or agents emerging observable collective behavior recognized as social phenomena (cf. Coleman, 1990: 6-10). This is probably a crucial point distinguished them to the study of psychology (or evolutionary psychology) that focus most of the observables in individuals constituting the social system.

The computational simulation based on the model proposed in Situngkir (2007), however, in such a way bridges the issue of the emergence and the non-linear nature of the micro- and the corresponding macro-properties. By adjusting the variables denoting the micro-system of social agent's cognitive system of sexual preferences, we grow the "living" structure in computer and dynamically we could observe the bird's eye view of the approached social system. If the micro-behavior of the social system is constructed in the fuzzy approach as described before, we could analyze the global properties, e.g.: the growth rate of the artificial system, the wealth distribution, and sometimes some analytical spectacles in observing the correlations among micro-system in the sense of human sexuality and the mating game.

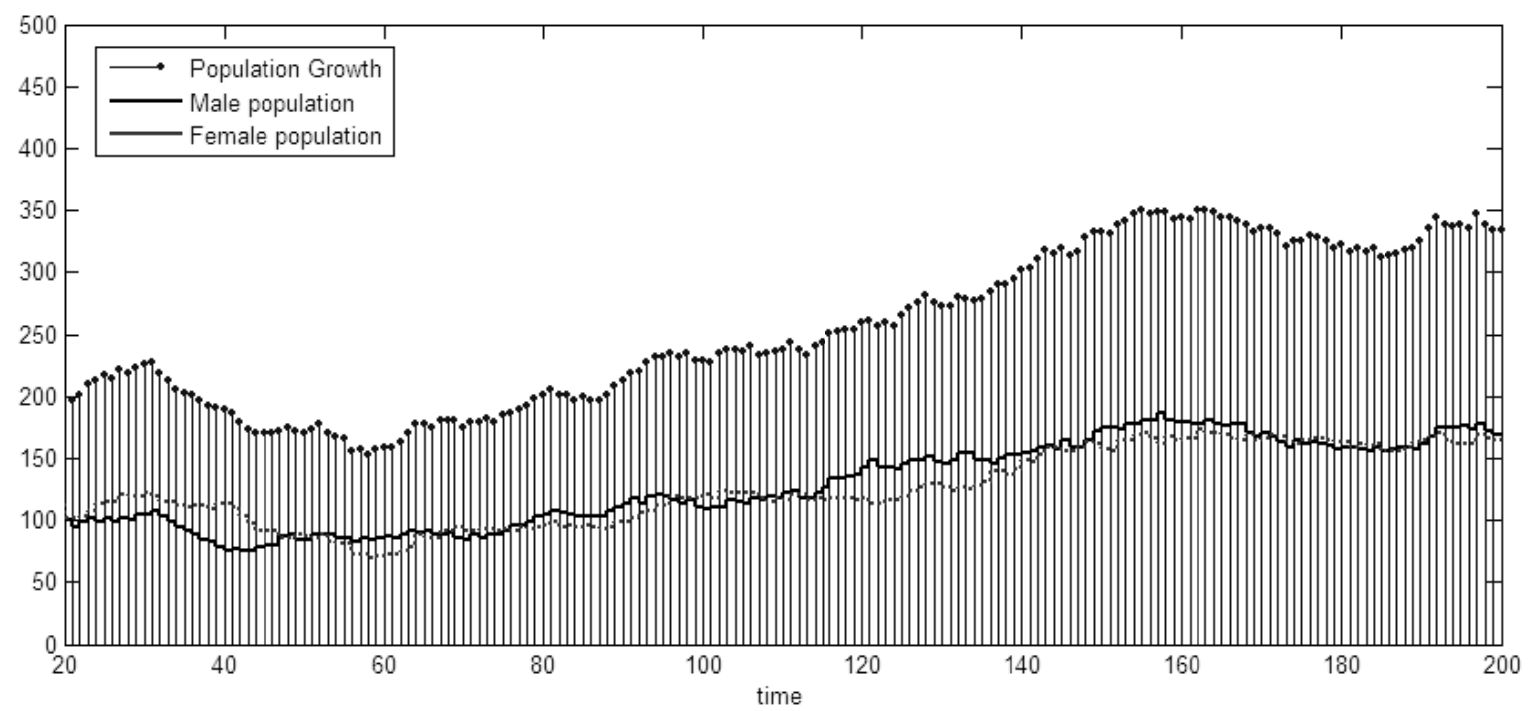

Figure 2. The computational simulation shows the dynamic equilibria of population growth in the Ribbon of Love.

Figure 2 shows how the computational simulation yields the equilibria of the population growth as well as the specific quantitative growth rate of both opposite sexes in the artificial world. However, we could see a lot more things in the simulation, as depicted 
in figure 3. This figure shows the correlation that could be captured in the simulation between the agents with high competitiveness in the perspective of their intelligence and the one in the eyes of physical performances. However, as the simulations conducted in figure 3 and 4 emerged from the "normal" micro properties of the system based on some findings in the observation in the field of evolutionary psychology, we can say that by learning through the resulting simulation, theoretically we could see that there is no exact correlations between intelligence and the physical outlook of the agents. By using the standard micro-properties of the artificial world, we could see that it is very rare to find an agent with significantly high attractiveness of mental intelligence $\left(q_{m \text { (intelligence) }}(t)\right)$ and simultaneously the most attractive physically $\left(Q_{p}(t)\right)$, also with the most attractive personal psychological traits $\left(q_{m(p)}(t)\right)$, e.g.: humor, romantic behavior to the opposite sex.
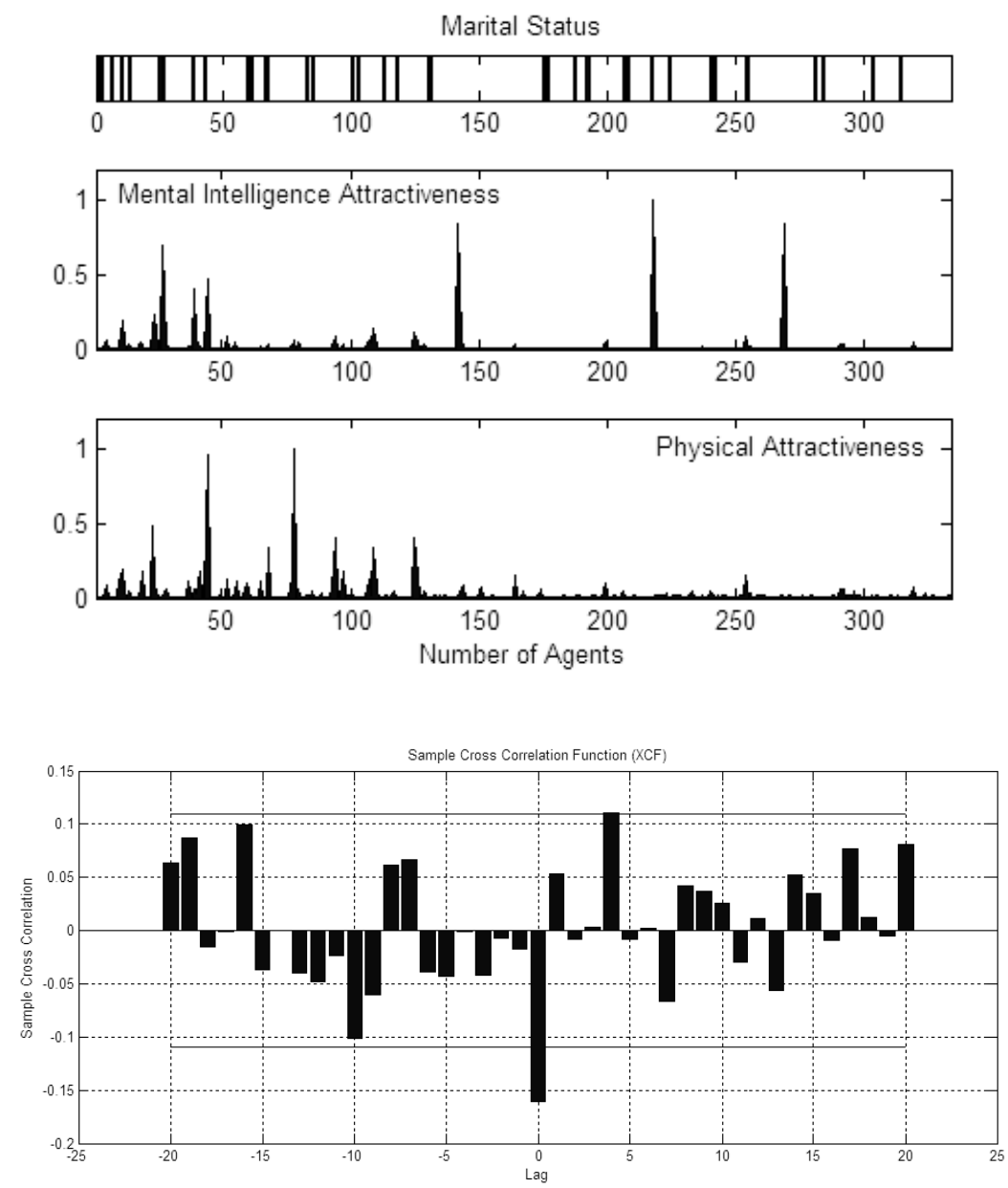

Figure 3. The attractiveness of a sexual agent in as represented in the attractiveness related to intelligence and physical appearance (above). The correlation coefficient of the $q_{m \text { (intelligence) }}(t)$ and $q_{m(p)}(t)$ as evaluated by corresponding mate candidates in the fuzzy fashion (below).

Is this some kind of accentuation to the broad commonsensical thoughts about this issue? Some further empirical works could be conducted in order to support this claim for it 
is presented quite strong in our simulation. Nonetheless, we must hold on to the clear definition of the attractiveness of intelligence and the attractiveness of physical performance, and it should not be corrupted to the generalization that it is impossible to find an intelligent and a good looking social agent in the real life. It is worth to note that the two variables are those that are related to the way how an opposite sex attracted to a respective agent and not merely an evaluation to a sole agent.

\section{Sexual Selection Shapes the Economy}

Another interesting findings from our simulations as we do several experiments in the computational platform is the emergence of the Pareto's power law distribution as we observe the macro properties after the few hundreds of our simulation. The initial condition of the system is made up by both uniformly and Gaussian $(N(\mu, \sigma))$ distributed wealth among all of the agents while there is no any economical activities regarding to agents' activities but the mating choice processes. There have been numerous empirical articles and papers showing this fact, but most of the works eventually relating the fact to the agent's economic micro-behavior and micro-motives. Nonetheless, our simulation showed that such economical macro-properties of the social system are emerged giving us theoretical conjectures that the scaling behavior of economic system is also shaped not solely by human motives and the natural (positive) feedback of the "networking" the richest with the richest yielding the richest gets richer. Furthermore, sexual selection among human bounded by the specific cultural constraints has become a natural factor emerging such interesting phenomena.

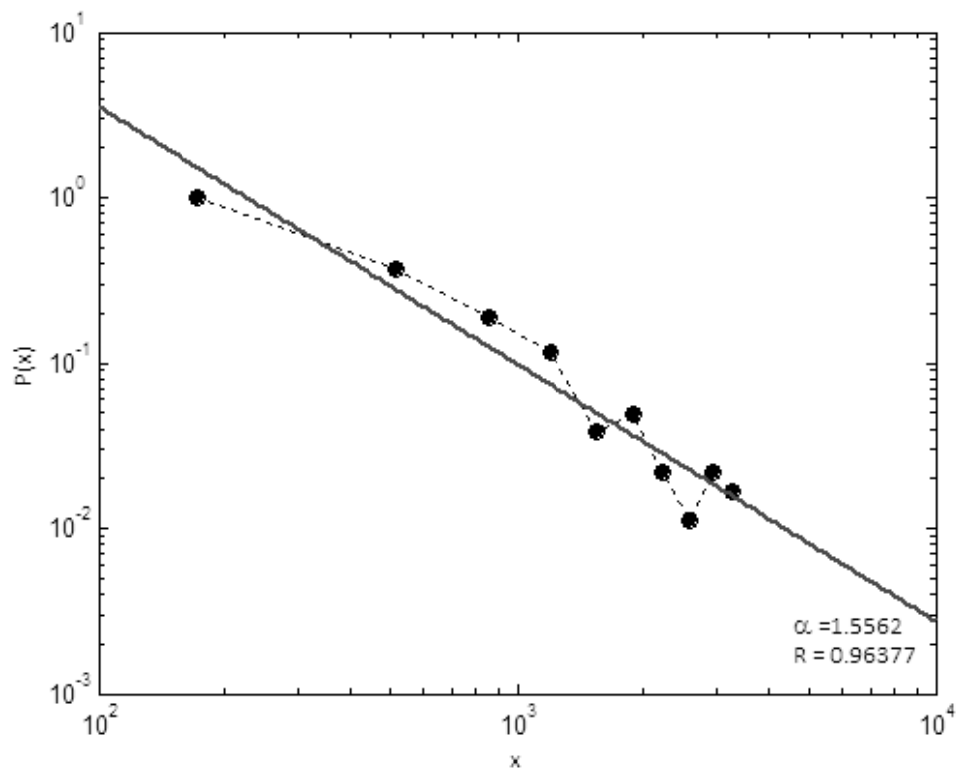

Figure 4. The Paretian scaling behavior of the distribution of wealth as shaped by the sexual selection processes.

In our case, the way agents choose the best available and accessible mating candidate probably become the source of the positive feedback fulfilling this Paretian 
wealth distribution. Furthermore, we also should note that the way an agent chooses other agents via the economic situation of individuals is not directly at all. In return, the wealthier an agent the higher his/her possibilities to become smarter $\left(q_{m \text { (intelligence) }}(t)>>\right)$ as well as to be better in physical outlook, $q_{m(p)}(t)>>$ (the opportunity to have better complexions, body building or skin treatment, etc.). Nonetheless, this scaling feature is still interesting for none economic motives and expectation directly included in an agent's decision making.

Learning from the properties of the power law as discussed in detail by Newman (2005), we can see that the expected maximum value in our samples would fulfill,

$<X_{\max }>\sim n^{\frac{1}{(\alpha-1)}}$

and as $2<\alpha<1$, we could see that $<x_{\max }>$ would diverge and by regarding to the mean of the power law characteristic as $n \rightarrow \infty$, the mean is finite but dominated by the largest value in the sample. Here, we can realize that as the sample data grows larger, the wealthiest agents in our Ribbon of Love rule relatively almost all of the economic system. A widely-accepted understanding on romance and love story brought to us the dominance of the richer among other population.

\section{Love, Infidelity, Marriage Liberalism}

In our previous computational experiments we assume that agents would always be faithful with their spouses: there is no way there would be any possibilities an agent flirt with other agent as they are in the state of marriage couples. However, what happens in the real world is never like that, especially when the self-centered society and marriage or sexual-related relations is relatively not really a sacred things. This distinction can be observed in the more religious society which the traditional understanding about marriage and sex as a kind of taboo like Indonesia.
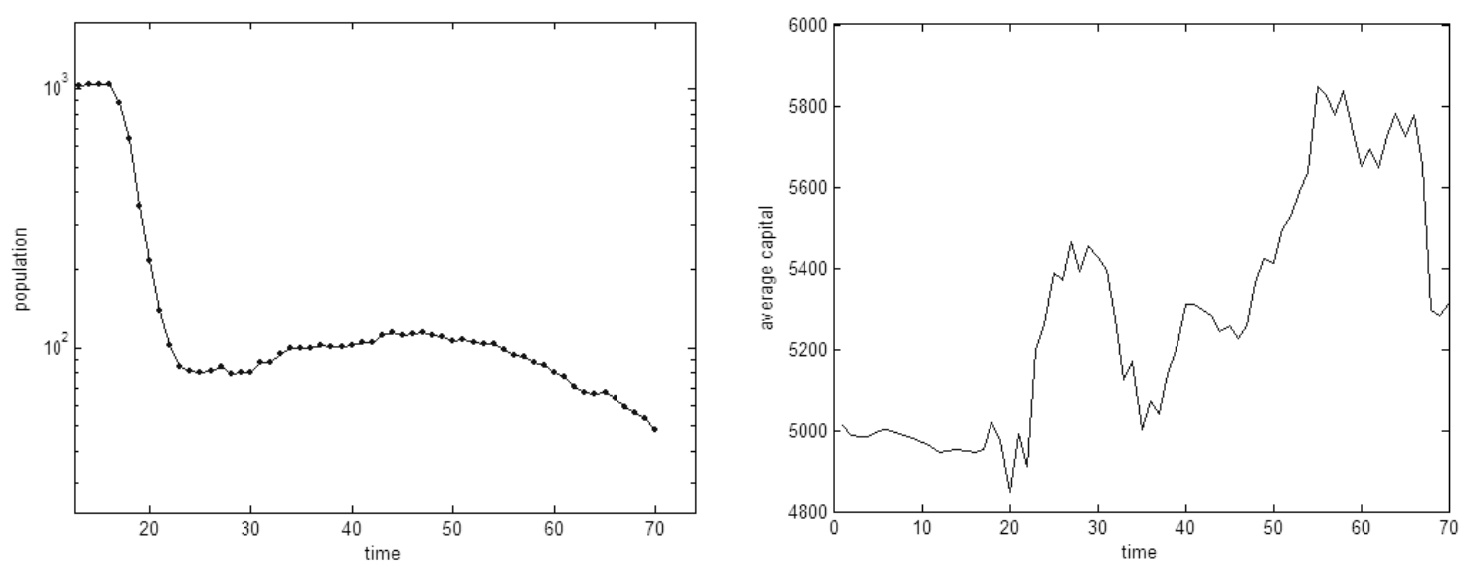

Figure 5. The negative population growth (left) and higher average economic growth (right) when the shortterm and choosier agents dominate. 
What happens in our artificial society while we gave up the assumption of the one marriage for the whole life in the sense of agents pursue better mates in short term relationship (cf. Buss, 1993)? Interestingly we could see a drastic drop of the population growth: the negative population growth over time. In contrast, the average agent's economic circumstances (represented by the average capital owned by agents) are better in the long run of the simulation. This is things that we could see in the more liberal society in which social actors saw marriage is relatively not really a sacred thing while there is no social pressure to keep the marriage goes on as some personal obstacles persist.

The sexual competition is apparently higher since agents become choosier as they always look on the betterment of the relationship with other persons comparatively, sexual relationship is related to fun much relative to as a part of institution of the reproduction. Obviously, such micro-structure is not easy to find relatively in the places where the debates between pro-life and pro-choice sex are rarely on the stage. Interestingly, the general economic conditions between the two extremes places on which we laid our two poles of assumptions are also distinctive.

Apparently, the better general economic a condition in the more liberal society is somehow could be related to the issue on how agents perceive the notions of sacred marriage for which the pro-life sex dominates most. However, we could apparently see that the economic growth of a society is not as simple as thought by economists. The cultural effects related to one thing, i.e.: how agents perceive marriage are things that should be put into account to see those phenomena. Here, obviously this cultural situation can play a role as one from a lot other possible ways, to be the positive feedback shaping economic dimension of the social life.

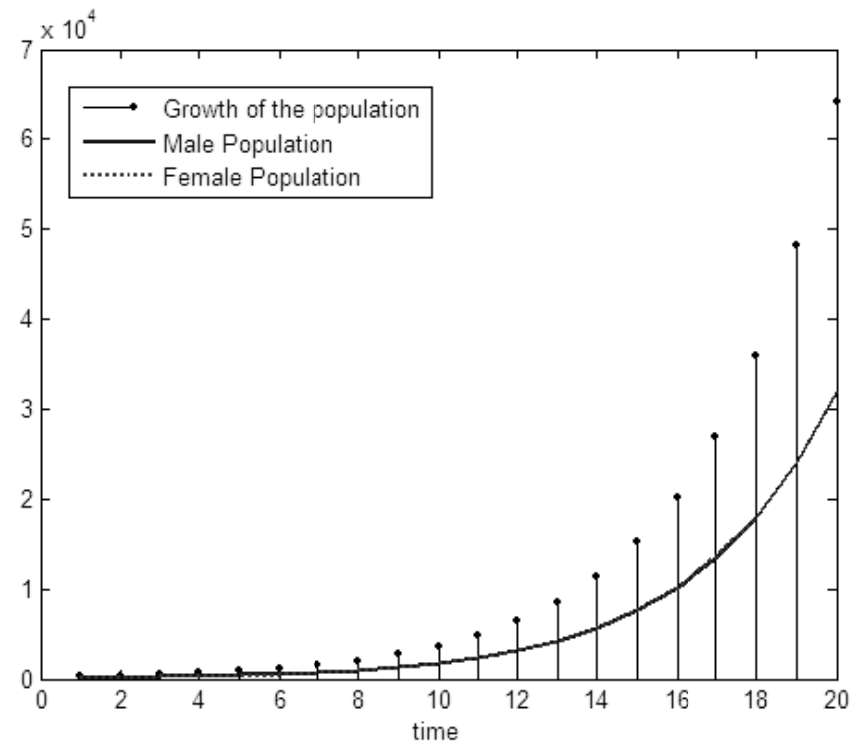

Figure 6. The exponential growth of population as we give possibilities to agents to choose the mates out of the fuzzification of the common sexual qualities. 


\section{If Love is Blind}

Liberally speaking we shall never be able to define what love is without being slipped into the reduction of one of important concept in human societies. Love may arise from the socially constructed values - like most liberal's believed - but may also arise from biological (or chemical?) processes among couples (Marazziti, 2004). Probably the concept of love is the place of the complexity as we talked about the human sexual selection.

In our previous experiments, as observer we feel to know why someone loves another, why someone wants to marry and start a family with another, as we reduce a lot of semantic thoughts on love. This section, eventually, wants to see what happens in our artificial society as we let agents chooses her/his spouses irrationally beside those with fuzzifications of some common sexual qualities.

While the other micro-properties are just similar with those elaborated in the generic computational model (Situngkir, 2007), we let agents to choose his/her mates in a particularly small probability $(p \approx 0.1$ ) and see the dynamics of the macro properties. To our interest, the growth of the population becomes exceedingly higher as the iteration goes. This is showed in figure 6 . There has been classical understanding of Malthusian economics that the population growth in approximately exponential function if there is no any social mechanisms to reduce the rate of birth. In the other hand, some societies tend to see the proper and improperness in the marital status of adult males (and frequently females). In some cases, this becomes the source of the irrationality of the mating couple in our experiments. Eventually, as there is no birth control mechanisms included, even a very small probability of this may eventually lead to the exponential population growth.

Apparently, in our simulation of this particular scenario we do not put into account the limits of environmental factors e.g.: the availability of resources (for example food, jobs, lands for housing, etc.) that sometimes become natural things as the proponents for birth control. For instance, the use of contraception is not the only thing to reduce the population growth but agent's realization on career, the financial constraints, etc. and many other social, economic, and cultural related things must be put into account as a social actor start to establish family especially deciding to have children.

\section{Discussions \& Closing Notes}

The fuzziness of human male and female relationship has been discussed in Situngkir (2007) and the paper is a report on findings and conducted experiments by using the proposed model. We do some experiments by using scenarios to observe what would emerge from specific micro-states in the level of agency. The primary point of the paper is to show that by using the computational model of artificial societies we could easily observe the relations between different levels of description that is non-linear in its nature. It becomes possible to relate the population growth, the dynamics of economic welfare, and so on by adjusting some micro-properties and micro-rules related to sexual selection in human civilizations. However, in this sense, there are a lot more things could be observed by using this computational tool in our endeavor understanding the intra-species sexual selection with those some macro and sophisticated aspects of human culture.

The secondary points are highlighted from the simulations as we employ several scenarios showed in the paper. The way we discover some economic aspects e.g.: the power-law distributed of wealth in society, the paradoxical findings on the sacred values of 
marriage as perceived by agents in the modern micro-realms emerging the high numbers of domestic partnership instead of marriage and reproduction, pursuing of fun through sexual experiences, as the negative population growth persists while in the other hand the average economic circumstances getting better generally, are some of those that we could essentially observe by using the computational platform. Here we open a possible interesting challenge on bridging the evolutionary theories and the general sociological explorations. As we recognize the complexity of the social and natural system, we just consequently recognize the complexity inside the way men and women mate.

\section{Acknowledgement}

I thank Surya Research International for financial support.

\section{References}

Buss, D. M. (1986). “Preferences in Human Mate Selection”. Journal of Personality and Social Psychology 50 (3): 559-70.

Buss, D. M. (1993). "Sexual Strategies Theory: An Evolutionary Perspective on Human Mating". Psychological Review 100 (2): 204-32.

Coleman, J. S. (1990). Foundations of Social Theory. The Belknap Press of Harvard UP.

Hill, S. E., Reeve, H. K. (2004). “Mating Games: The Evolution of Human Mating Transactions”. Behavioral Ecology 15 (5): 748-56.

Holmström, K., Jensen, H. J. (2004). "Who Runs Fastest in an Adaptive Landscape: Sexual versus Asexual Reproduction". Physica A 337: 185-95.

Khanafiah, D., SItungkir, H. (2004). "Social Balance Theory: Revisiting Heider's Balance Theory for Many Agents". BFI Working Paper Series WPN2004. Bandung Fe Institute.

Liao, X., \& Ran, J. (2007). "Hopf Bifurcation in Love Dynamical Models with Nonlinear Couples and Time Delays". Chaos, Solitons, and Fractals 31: 853-65.

Marazziti, D., Canale, D. (2004). “Hormonal Changes when Falling in Love”. Psychoneuroendocrinology 29: 931-36.

Miller, G. F. (2000). The Mating Mind: How Sexual Choice Shaped the Evolution of Human Nature. Doubleday.

Rinaldi, S. (1998). “Love Dynamics: The Case of Linear Couples”. Applied Mathematics and Computation 95: 181-92.

Shackelford, T. K., Schimtt, D. P., Buss, D. M. (2005). “Universal Dimensions of Human Mate Preferences". Personality and Individual Differences 39: 447-58.

Situngkir, H. (2003). "Cultural Studies through Complexity Sciences: Beyond Postmodern Culture without Postmodern Theorists". BFI Working Paper Series WPM2003. Bandung Fe Institute.

Situngkir, H. (2004). "On Selfish Meme: Culure as Complex Adaptive System”.Journal of Social Complexity 2 (1): 20-32.

Situngkir, H. (2007). "The Ribbon of Love: Fuzzy-Ruled Agents in Artificial Society”. BFI Working Paper Series WPB2007.

van den Berghe, Pierre L. (1990). "Why most sociologists don't (and won't) think evolutionarily". Sociological Forum, 5, 173-185.

Strogatz, S. H. (1988). "Love Affairs and Differential Equations". Mathematics Magazine 61 (1): 35. 